\title{
THE DIFFERENTIAL TRANSFORMATION METHOD AND PADE APPROXIMANT FOR A FORM OF BLASIUS EQUATION
}

\author{
Haldun Alpaslan Peker, Onur Karaoğlu and Galip Oturanç \\ Department of Mathematics, Faculty of Science \\ Selçuk University, 42003 Konya, Turkey \\ pekera@hotmail.com
}

\begin{abstract}
Boundary conditions in an unbounded domain, i.e. boundary condition at infinity, pose a problem in general for the numerical solution methods. The aim of this study is to overcome this difficulty by using Padé approximation with the differential transform method (DTM) on a form of classical Blasius equation. The obtained results are compared with, for the first time, the ones obtained by using a modified form of Adomian decomposition method (ADM). Furthermore, in order to see the consistency of solutions, they are also compared with the ones obtained by using variational iteration method (VIM).
\end{abstract}

Key Words- Blasius equation, Padé Approximants, Differential Transformation Method (DTM), Boundary Layers

\section{INTRODUCTION}

In many areas of scientific fields such as plasma physics, fluid mechanics and population models, many of the nonlinear phenomena can be modeled by nonlinear differential equations. The nonlinear differential equations which have boundary conditions in unbounded domains have a great interest and they will be examined in this work. However, many of the modeled nonlinear equations do not have an analytical solution. Both analytical solutions methods and numerical solutions methods are used to solve these equations. For instances, Adomian decomposition method [1-3], homotopy perturbation method [3, 4], variational iteration method $[5,6,24]$. The differential transform method [7-15] is one of the effective and reliable numerical solution methods for handling both linear and nonlinear differential equations.

In this work, the differential transform method will be applied to a form of classical Blasius equation $[16,17]$.

\section{BLASIUS EQUATION}

Many different but related phenomena are stated and studied by the Blasius equation $[9,18,19]$ that has a special importance for all boundary-layer equations in fluid mechanics. The Blasius equation can be described as the non-dimensional velocity distribution in the laminar boundary layer over a flat plate which is shown in Fig.1. 

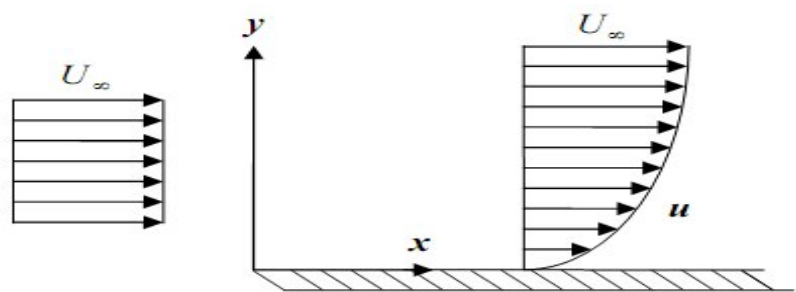

Figure 1. Laminar flow over a flat plate

The continuity equation, the Navier Stokes equation and the boundary conditions can be expressed as [20]:

$\frac{\partial u}{\partial x}+\frac{\partial u}{\partial y}=0$

$u \frac{\partial u}{\partial x}+v \frac{\partial u}{\partial y}=v \frac{\partial^{2} u}{\partial y^{2}}$

at $y=0: \quad u=v=0$,

as $y \rightarrow \infty: u \rightarrow U_{\infty}$,

at $x=0: \quad u=U_{\infty}$,

where $u$ and $v$ are the velocity in the $x$ and $y$ direction respectively.

After a group of transformations [20], the equations (1-5) reduce to the following form:

$$
\begin{aligned}
& f^{\prime \prime \prime}(\eta)+\frac{1}{2} f(\eta) f^{\prime \prime}(\eta)=0 \\
& f(0)=0, f^{\prime}(0)=0, f^{\prime}(\infty)=1,0<\eta<\infty .
\end{aligned}
$$

However, Eq. (6-7) and other forms of Blasius problem have boundary condition in unbounded domains, i.e. boundary condition at infinity and this makes a difficulty for the numerical solution methods. To overcome this difficulty, Padé approximants can be applied to manipulate the obtained series for numerical approximations. For instance, in [21], using Padé approximants, Thomas-Fermi equation, which also contains boundary condition at infinity, was solved by the differential transform method.

\section{DIFFERENTIAL TRANSFORMATION METHOD}

Differential transform method is a numerical method based on Taylor expansion. This method tries to find coefficients of series expansion of unknown function by using the initial data on the problem. The concept of differential transform method was first proposed by Zhou [7]. It was applied to electric circuit analysis problems by Zhou [7]. Afterwards, it was applied to several systems and differential equations. For instance, initial - value problems [10], difference equations [11], integro-differential equations [12], partial differential equations $[13,14]$, system of ordinary differential equations [15].

Definition 1. The one-dimensional differential transform of a function $y(x)$ at the point $x=x_{0}$ is defined as follows $[8,15]$ : 


$$
\mathrm{Y}(\mathrm{k})=\frac{1}{\mathrm{k} !}\left[\frac{\mathrm{d}^{\mathrm{k}}}{\mathrm{dt}^{\mathrm{k}}} \mathrm{y}(\mathrm{x})\right]_{\mathrm{x}=\mathrm{x}_{0}},
$$

where $y(x)$ is the original function and $Y(k)$ is the transformed function.

Definition 2. The differential inverse transform of $Y(k)$ is defined as follows [8,15]:

$$
\mathrm{y}(\mathrm{x})=\sum_{\mathrm{k}=0}^{\infty} \mathrm{Y}(\mathrm{k})\left(\mathrm{x}-\mathrm{x}_{0}\right)^{\mathrm{k}} .
$$

From (8) and (9) we obtain

$$
\mathrm{y}(\mathrm{x})=\sum_{\mathrm{k}=0}^{\infty} \frac{1}{\mathrm{k} !}\left[\frac{\mathrm{d}^{\mathrm{k}}}{\mathrm{dt}^{\mathrm{k}}} \mathrm{y}(\mathrm{x})\right]_{\mathrm{x}=\mathrm{x}_{0}}\left(\mathrm{x}-\mathrm{x}_{0}\right)^{\mathrm{k}} .
$$
below:

The following theorems that can be deduced from Defs. 3.1. and 3.2. are given

Theorem 1. If $f(x)=g(x) \pm h(x)$, then $F(k)=G(k) \pm H(k)$.

Theorem 2. If $f(x)=\lambda g(x)$, then $F(k)=\lambda G(k)$, where $\lambda$ is a constant.

Theorem 3. If $f(x)=\frac{d^{n} g(x)}{d x^{n}}$, then $F(k)=\frac{(k+n) !}{k !} G(k+n)$.

Theorem 4. If $f(x)=g(x) h(x)$, then $F(k)=G(k) \otimes H(k)=\sum_{k_{1}=0}^{k} G\left(k_{1}\right) H\left(k-k_{1}\right)$.

Theorem 5. If $f(x)=g(x) \frac{d^{2} h(x)}{d x^{2}}$, then $F(k)=G(k) \otimes P(k)=\sum_{r=0}^{k}(k-r+1)(k-r+2) F(r) P(k-r+2)$.

\section{PADE APPROXIMANT}

A well known fact is that polynomials are used to approximate truncated power series. Further, the singularities of polynomials cannot be seen obviously in a finite plane. Since the radius of convergence of the power series may not large enough to contain the two boundaries, it is not always useful to use the power series [22]. Padé approximants are applied to manipulate the obtained series for numerical approximations to overcome this difficulty. Padé approximant is the best approximation of a polynomial approximation of a function into rational functions of polynomials of given order [23].

Padé approximants are used widely in computer calculations due to the fact that a Padé approximant often gives better approximation of the function than truncating its power series and it may still work where the power series does not converge. Therefore, Padé approximants can be easily computed by using symbolic programming languages such as Maple or Mathematica.

A Padé approximation to $f(x)$ on $[a, b]$ is the quotient of two polynomials, say $P_{N}(x)$ and $Q_{M}(x)$ of degrees $N$ and $M$ respectively [23]. The notation $[N / M]$ will be used to denote this quotient. 
In order to obtain better numerical results, the combination of the differential transform method and the diagonal approximants $[N / N]$ will be used.

\section{APPLICATION}

Consider the following boundary value problem related to another form of classical Blasius problem [16, 17].

$$
\begin{aligned}
& f^{\prime \prime \prime}(\eta)+\frac{1}{2} f(\eta) f^{\prime \prime}(\eta)=0 \\
& f(0)=0, f^{\prime}(0)=1, f^{\prime}(-\infty)=0,-\infty<\eta<0 .
\end{aligned}
$$

In order to find numerical values of $f^{\prime \prime}(0)$, the series obtained by the differential transform method and the diagonal Padé approximants will be combined. Several diagonal Padé approximants of different degrees will be used to form an opinion about the behavior of the solution of the Blasius equation.

Note that $f^{\prime \prime}(0)=A$, where $A$ is a positive constant.

Now, the differential transform method will be applied to (11) as follows;

$$
(k+1)(k+2)(k+3) F(k+3)+\frac{1}{2} \sum_{r=0}^{k}(k-r+1)(k-r+2) F(r) F(k-r+2)=0
$$

It is also necessary to find differential transform equivalence form of initial and boundary values. For the initial and boundary values, differential transform equivalences are as follows;

$$
F(0)=0, F(1)=1, F(2)=\frac{A}{2}
$$

Using Maple, from (13) and (14), the following solution is obtained;

$$
\begin{aligned}
f(\eta)= & \eta+\frac{A}{2} \eta^{2}-\frac{A}{48} \eta^{4}-\frac{A^{2}}{240} \eta^{5}+\frac{A}{960} \eta^{6}+\frac{11 A^{2}}{20160} \eta^{7}+\left(-\frac{1}{21504} A+\frac{11}{161280} A^{3}\right) \eta^{8}-\frac{43 A^{2}}{967680} \eta^{9} \\
& +\left(\frac{A}{552960}-\frac{5}{387072} A^{3}\right) \eta^{10}+\left(\frac{221 A^{2}}{106444800}-\frac{29 A\left(-\frac{1}{21504} A+\frac{11}{161280} A^{3}\right)}{1980}-\frac{A^{4}}{570240}\right) \eta^{11} \\
& +\left(-\frac{1}{16220160} A+\frac{1}{725760} A^{3}\right) \eta^{12}+\ldots
\end{aligned}
$$
condition

Now, our aim is to determine a numerical value for $A$ using the boundary

$$
\lim _{\eta \rightarrow-\infty} f^{\prime}(\eta)=0
$$

which is in condition (12). In order to do this, derivative of polynomial solution (15) should be taken. Then, after the formation of this equation by Padé approximation, we will apply the condition (16) for the obtained rational function.

$$
\lim _{\eta \rightarrow-\infty} \frac{3\left(4+3 A x+\left(-A^{2}+\frac{1}{3}\right) x^{2}\right)}{12-3 A x+x^{2}}=-3 A^{2}+1 \text { for }[2 / 2] \text {. }
$$


At last, the value $A=0.5773502693$ is obtained from equation $-3 A^{2}+1=0$. $\lim _{\eta \rightarrow-\infty} \frac{300 A^{2}-40+\left(300 A^{3}-70 A\right) x-3 x^{2}+\left(\frac{45}{4} A^{3}-3 A\right) x^{3}}{10\left(30 A^{2}-4-3 A x+\left(-\frac{3}{10}+3 A^{2}\right) x^{2}+\left(\frac{5}{8} A^{3}-\frac{1}{3} A\right) x^{3}\right)}=\frac{9\left(15 A^{2}-4\right)}{5\left(15 A^{2}-8\right)}$ for $[3 / 3]$.
At last, the value $A=0.5163977795$ is obtained from equation $\frac{9\left(15 A^{2}-4\right)}{5\left(15 A^{2}-8\right)}=0$. $\lim _{\eta \rightarrow-\infty} \frac{225 A^{4}+60 A^{2}-26+\left(225 A^{5}-\frac{225}{4} A^{3}+\frac{17}{2} A\right) x+\left(-\frac{690}{7} A^{4}+\frac{2619}{56} A^{2}-\frac{39}{7}\right) x^{2}+\left(\frac{135}{28} A^{5}-\frac{99}{56} A^{3}-\frac{69}{112} A\right) x^{3}+\left(\frac{135}{112} A^{6}-\frac{189}{64} A^{4}+\frac{51}{28} A^{2}-\frac{169}{560}\right) x^{4}}{225 A^{4}+60 A^{2}-26+\left(-\frac{465}{4} A^{3}+\frac{69}{2} A\right) x+\left(\frac{495}{28} A^{4}+\frac{687}{56} A^{2}-\frac{39}{7} A\right) x^{3}+\left(\frac{165}{28} A^{5}-\frac{253}{28} A^{3}+\frac{937}{336} A\right) x^{3}+\left(-\frac{1055}{448} A^{4}+\frac{153}{112} A^{2}-\frac{169}{560}\right) x^{4}}$ $=-\frac{2700 A^{6}-6615 A^{4}+4080 A^{2}-676}{5275 A^{4}-3060 A^{2}+676}$ for $[4 / 4]$.

At last, the value $A=0.5227030798$ is obtained from equation $-\frac{2700 A^{6}-6615 A^{4}+4080 A^{2}-676}{5275 A^{4}-3060 A^{2}+676}=0$.

In [16], Padé calculations were made utmost [4/4] diagonal approximant. Hence, we will make our comparisons till this value.

The diagonal approximants [2/2], [3/3] and [4/4] were computed by using Maple 12. According to the above procedure, obtained values for $A$ are listed at the left column in Table 1 below. Besides this, at the right column of the Table 1, the obtained values for $A$ by using a modified form of Adomian decomposition method in [16] are listed.

Table 1. Padé approximants and numerical value of $A$

\begin{tabular}{|c|c|c|}
\hline Padé Approximant & $A$ & $A$ (Ref. [16]) \\
\hline$[2 / 2]$ & 0.5773502693 & 0.5773502693 \\
\hline$[3 / 3]$ & 0.5163977795 & 0.5163977793 \\
\hline$[4 / 4]$ & 0.5227030798 & 0.5227030798 \\
\hline
\end{tabular}

In addition to these, there does not exist analytical solution for this problem. Therefore, in order to see the consistency of solutions, the series solution up to the $12^{\text {th }}$ term of the problem are calculated by the differential transform method, Adomian decomposition method and variational iteration method respectively with respect to the numerical value of $A$ for the [4/4] diagonal approximant for which 0.5227030796 is the obtained value by variational iteration method [24]. Furthermore, they are calculated with step size 0.1 on the interval $[0,1]$ and the results are shown graphically in Figure 1 below. 


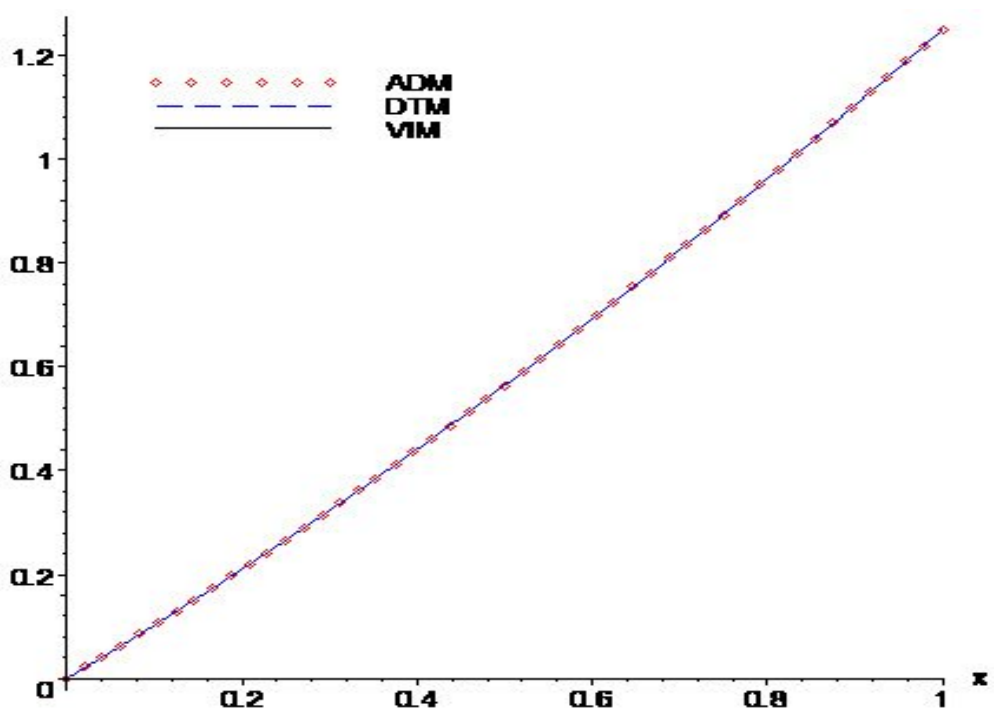

Figure 1. Results obtained by ADM, DTM and VIM

\section{CONCLUSION}

Boundary conditions in an unbounded domain, i.e. boundary condition at infinity, pose a problem in general for the numerical solution methods. In this work, in order to get ride off this problem, Padé approximation with the differential transform method was used. The computations done above were performed by using Maple 12 .

The results we obtained in this study by the differential transform method are compared with, for the first time, the ones found by using a modified form of Adomian decomposition method in [16], which can be easily seen in Table 1.

Finally, convergence of the obtained results can also be seen from Figure 1. The results obtained here are in accord with the one found by variational iteration method, i.e. all of them are coincides.

Acknowledgement-This study was supported by the Coordinatorship of Selçuk University's Scientific Research Projects (BAP).

\section{REFERENCES}

1. G. Adomian, A review of the decomposition method in applied mathematics, J. Math. Anal. Appl. 135, 501-544, 1988.

2. S. A. Khuri, On the decomposition method for the approximate solution of nonlinear ordinary differential equations, Int. J. Math. Educ. Sci. Technol. 32, 525-539, 2001.

3. S. Abbasbandy, A numerical solution of Blasius equation by Adomian's decomposition method and comparison with homotopy perturbation method, Chaos, Solitons and Fractals 31, 257-260, 2007.

4. J. H. He, Homotopy perturbation method: a new nonlinear technique, Appl. Math. Comput. 135, 73-79, 2003.

5. J. H. He, A new approach to nonlinear partial differential equations, Comm. Nonlinear Sci. Numer. Simul. 2, 203-205, 1997.

6. J. H. He, A variational iteration approach to nonlinear problems and its applications, Mech. Appl. 20, 30-31, 1998. 
7. J.K. Zhou, Differential Transformation and its Applications for Electrical Circuits, Huarjung University Press, Wuuhahn, China, 1986.

8. I.H. Abdel-Halim Hassan, Different applications for the differential transformation in the differential equations, Applied Mathematics and Computation 129, 183-201, 2002.

9. A. Arıkoğlu and İ. Özkol, Inner-outer matching solution of Blasius equation by DTM, Aircraft Engineering and Aerospace Technology: An International Journal 77, 298-301, 2005.

10. M.-J. Jang, C.-L. Chen and Y.-C. Liy, On solving the initial-value problems using the differential transformation method, Applied Mathematics and Computation 115, 145-160, 2000.

11. A. Arıkoğlu and İ. Özkol, Solution of difference equations by using differential transform method, Applied Mathematics and Computation 174, 1216-1228, 2006.

12. P. Darania and A. Ebadian, A method for the numerical solution of the integrodifferential equations, Applied Mathematics and Computation 188, 657-668, 2007.

13. A. Kurnaz, G. Oturanç and M. E. Kiriş, n-Dimensional differential transformation method for solving PDEs, International Journal of Computer Mathematics 82, 369-380, 2005.

14. F. Ayaz, On the two-dimensional differential transform method, Applied Mathematics and Computation 143, 361-374, 2003.

15. A. Kurnaz and G. Oturanç, The differential transform approximation for the system of ordinary differential equations, International Journal of Computer Mathematics 82, 709-719, 2005.

16. A. Wazwaz, A reliable algorithm for solving boundary value problems for higherorder integro-differential equations, Applied Mathematics and Computation 118, 327 $342,2001$.

17. A. Wazwaz, The modified decomposition method and Padé approximants for a boundary layer equation in unbounded domain, Applied Mathematics and Computation 177, 737-744, 2006.

18. T. Fang, W. Liang and C. F. Lee, A new solution branch for the Blasius equation-A shrinking sheet problem, Computers \& Mathematics with Applications 56, 3088-3095, 2008.

19. T. Fang and C. F. Lee, A moving-wall boundary layer flow of a slightly rarefied gas free stream over a moving flat plate, Applied Mathematics Letters 18, 487-495, 2005.

20. B.-L. Kuo, Thermal boundary-layer problems in a semi-infinite flat plate by the differential transformation method, Applied Mathematics and Computation 150, 303320, 2004.

21. G. Oturanç, A new approach to the Thomas-Fermi equation, Selcuk Journal of Applied Mathematics, 10, 67-74, 2009.

22. J. Boyd, Padé approximant algorithm for solving nonlinear ordinary differential equation boundary value problems on an unbounded domain, Comput. Phys. 11, 299303, 1997.

23. G. A. Baker and P. Graves-Morris, Padé Approximants, Cambridge U.P., 1996.

24. Abdul-Majid Wazwaz, The variational iteration method for solving two forms of Blasius equation on a half-infinite domain, Applied Mathematics and Computation, 188, 485-491, 2007. 